\title{
3D-printed resonant gold nanocones for out-of-plane terahertz-field-driven electron photoemission
}

\author{
Andrea Rovere ${ }^{1}$, Riccardo Piccoli ${ }^{1}$, Andrea Bertoncini ${ }^{2}$, Young-Gyun Jeong ${ }^{1}$, Stéphane Payeur ${ }^{1}$, François \\ Vidal $^{1}$, O-Pil Kwon ${ }^{3}$, Seung-Heon Lee ${ }^{3}$, Roberto Morandotti ${ }^{1,4}$, Carlo Liberale ${ }^{2 *}$, Luca Razzari ${ }^{{ }^{*}}$ \\ ${ }^{1}$ Institut National de la Recherche Scientifique, Centre Énergie, Matériaux et Télécommunications, Varennes, Québec J3X 1S2, Canada \\ ${ }^{2}$ KAUST, BESE (Biological Environmental Science and Engineering) division, Thuwal 23955-6900, Saudi Arabia \\ ${ }^{3}$ Department of Molecular Science and Technology, Ajou University, Suwon 16499, Republic of Korea \\ ${ }^{4}$ Institute of Fundamental and Frontier Sciences, University of Electronic Science and Technology of China, Chengdu 610054, China \\ razzari@emt.inrs.ca carlo.liberale@kaust.edu.sa
}

\begin{abstract}
We numerically and experimentally investigate out-of-plane gold nanostructures resonating at terahertz frequencies for field-driven photoemission and compare their performance with a traditional non-resonant nanotip geometry. (C) 2020 The Authors
\end{abstract}

\section{Introduction}

Ultrashort electron bunches can be extracted from metallic nanotips by means of terahertz (THz)-field-driven electron tunneling [1,2]. Recent studies have suggested that resonant antennas can provide a higher local field enhancement and thus relax the requirement for extremely intense THz sources [3,4]. However, 2D planar antenna geometries, which have been investigated so far, still present limitations. For instance, besides typically featuring low quality factor resonances, the close proximity of the substrate as well as the in-plane direction of the resonating electric field hamper their effective use as photocathodes. Recent advances in nanofabrication technologies allow the exploration of complex 3D architectures, enabling not only the precise design of a single nanostructure, but also a full control in the arrangement of multiple antennas, so that their collective electromagnetic response can be precisely tailored. Here, we present an out-of-plane, resonant nanoantenna design for ultrafast field-driven photoemission, taking advantage of high-resolution 3D printing lithography [5]. In particular, we exploit the strong monopolar resonance of gold vertical nanocones (NCs), prepared on a conductive gold surface acting as a mirror-image plane (Figure 1a), to achieve a significant boost in field-driven electron emission in comparison with standard non-resonant nanotips. The collective electromagnetic response of the NC array can be engineered to obtain a constructive interference among the scattered fields from the individual NCs (through the exploitation of a "lattice mode" [6]), which results in a stronger local field enhancement and a narrower resonance bandwidth in the far field response. We show that the benefit offered by this "cooperative effect" can also be preserved for the case of a single NC surrounded by resonant cylinders (Figure 1b).

\section{Results and Discussion}

The design of the resonant structures has been optimized via numerical simulations using COMSOL Multiphysics, to achieve a monopole resonance at around $1 \mathrm{THz}$. All the samples have been fabricated via a high-resolution 3D printing technology (Nanoscribe) based on two-photon absorption in a polymer material, followed by the evaporation of a 200-nm-thick gold layer. The NCs present an aspect ratio of 1/10 (base diameter/height), with a tip radius of curvature of $150 \mathrm{~nm}$ (inset of Figure 1b). The $\mathrm{THz}$ response of the arrays has been measured in reflection using a $\mathrm{THz}$ time-domain spectroscopy setup, revealing a good agreement between simulation and experimental results (Figure 1c reports the case of a single NC in an array of cylinders, more details about other comparative samples can be found in [5]). As a first proof of electron photoemission, we have studied the electroninduced fluorescence of argon atoms in proximity of the $\mathrm{NC}$ tips, when excited by an organic-crystal-based $\mathrm{THz}$ source [7] (effective peak electric field of $\sim 80 \mathrm{kV} / \mathrm{cm}$ along the nanostructures main axis). The nonlinear relation between the recorded fluorescence intensity and the impinging $\mathrm{THz}$ peak electric field (see inset of Figure 1d for the case of the NC in an array of cylinders) can be described by the Fowler-Nordheim (FN) equation. By fitting the experimental data with such equation in a convenient logarithmic representation (Figure 1d), we have retrieved the time-domain field enhancement at the nanostructures apex, which is the key parameter to compare the performance of different photoemitters, achieving a value of 128 and 322 for the non-resonant nanotip and the NC in an array of cylinders, respectively. Furthermore, via a two-step model to describe the THz-induced electron extraction and subsequent acceleration, we have estimated an increase in the electrons energy cut-off of a factor of 2 (from 200 $\mathrm{eV}$ for the non-resonant case to $400 \mathrm{eV}$ for our optimized design). Finally, we have directly recorded (using an in- 
house built setup) the emitted electron current, which resulted to be reasonably stable in time. The structural stability of the fabricated samples has been further confirmed through SEM analysis after a prolonged $\mathrm{THz}$ exposure (>107 $\mathrm{THz}$ pulses).
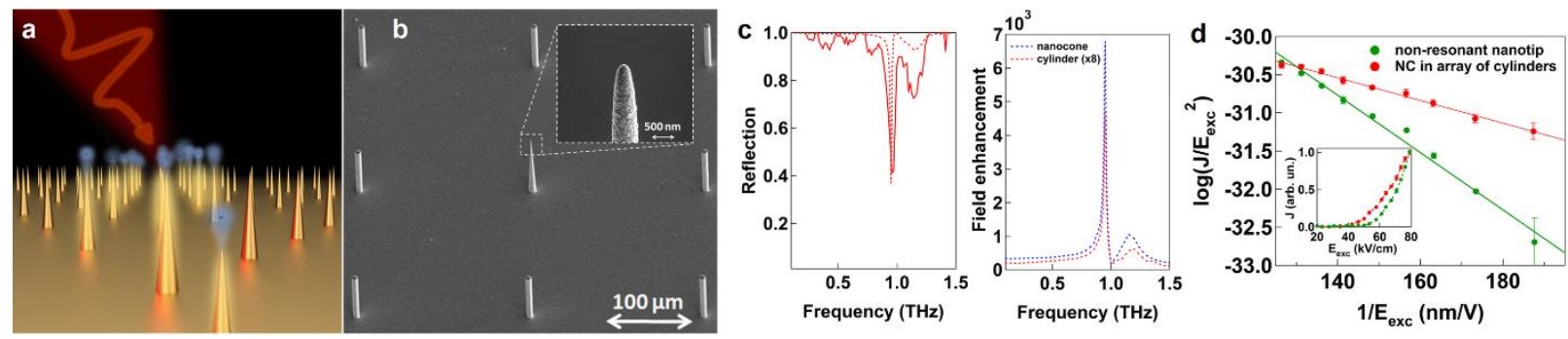

Fig. 1. a) Illustration of THz field-driven photoemission from an array of monopolar gold NCs. b) SEM image of the single NC in an array of cylinders. The details of the NC apex are shown in the inset. c) (left) numerical simulation and experimental characterization of the far-field reflection of the sample. (right) numerically evaluated field enhancement as a function of frequency at the NC apex (blue) and in proximity of the top of the cylinder (red). d) Experimental data (dots) of the measured argon fluorescence from the NC in an array of cylinders (red) and from the non-resonant nanotip (green), presented in a convenient logarithmic representation, allowing a linear fit with the FN equation (solid line). The directly measured fluorescence values (normalized to their respective maximum) as a function of the incident $\mathrm{THz}$ electric field are reported in the inset.

In summary, a novel resonant photocathode design for ultrafast THz-field-driven photoemission has been proposed for out-of-plane electron emission and acceleration employing table-top $\mathrm{THz}$ sources with peak electric fields at the $100 \mathrm{kV} / \mathrm{cm}$ level. A novel degree of optimization has been introduced, relying on tailoring the collective response of resonant structures when arranged in an array geometry. A boost of the nano-localized timedomain THz peak electric field of a factor of 2.5 when compared to a standard non-resonant nanotip has been found for the cooperative nanostructures, also for the case of a single nanoemitter in an array of cylinders. We stress that, while our strategy has been demonstrated for the case of a broadband $\mathrm{THz}$ source, the use of a narrowband $\mathrm{THz}$ source would grant access to the full exploitation of the sharp monopolar resonance of the NCs. Our approach may thus open the path for a new generation of photocathodes that can be designed at will and reproducibly fabricated via advanced 3D printing, significantly relaxing the requirement for intense $\mathrm{THz}$ drivers.

[1] G. Herink, L. Wimmer and C. Ropers, "Field emission at terahertz frequencies: AC-tunneling and ultrafast carrier dynamics", New J. Phys. 16, 123005 (2014).

[2] S. Li and R. R. Jones, "High-energy electron emission from metallic nano-tips driven by intense single-cycle terahertz pulses", Nature Commun. 7, 13405 (2016).

[3] J. Zhan, X. Zhao, K. Fan, X. Wang, G.-F. Zhang, K. Geng, X. Zhang and R. D. Averitt, "Terahertz radiation-induced sub-cycle field electron emission across a split-gap dipole antenna", Appl. Phys. Lett. 107, 231101 (2015).

[4] K. Iwaszczuk, M. Zalkovskij, A. C. Strikwerda and P. U. Jepsen, "Nitrogen plasma formation through terahertz-induced ultrafast electron field emission", Optica 2, 116 (2015).

[5] A. Rovere, R. Piccoli, A. Bertoncini, Y.-G. Jeong, S. Payeur, F. Vidal, S.-H. Lee, J.-H. Seok, O-P. Kwon, R. Morandotti, C. Liberale, L. Razzari, “3D-Printed Photocathodes for Resonant, Terahertz-Field-Driven Ultrafast Electron Emission”, ArXiv: 2010.12098 (2020).

[6] M. Malerba, A. Alabastri, E. Miele, P. Zilio, M. Patrini, D. Bajoni, G. C. Messina, M. Dipalo, A. Toma, R. Proietti Zaccaria and F. De Angelis, "3D vertical nanostructures for enhanced infrared plasmonics", Sci. Rep. 5, 16436 (2015).

[7] A. Rovere, Y.-G. Jeong, R. Piccoli, S.-H. Lee, S.-C. Lee, O.-P. Kwon, M. Jazbinsek, R. Morandotti and L. Razzari, “Generation of high-field terahertz pulses in an HMQ-TMS organic crystal pumped by an ytterbium laser at $1030 \mathrm{~nm}$ ”, Opt. Express 26, 2509-2516 (2018). 